\title{
An age-integrated approach to improve measurement of potential spatial accessibility to emergency medical services for urban areas
}

\author{
Soheil Hashtarkhani $^{1}$ (1) | Behzad Kiani ${ }^{1}$ | Robert Bergquist ${ }^{2}$ | \\ Nasser Bagheri ${ }^{3}$ | Reza VafaeiNejad ${ }^{4}$ | Mahmood Tara ${ }^{1}$
}

${ }^{1}$ Department of Medical Informatics, Mashhad University of Medical Sciences, Mashhad, Iran

${ }^{2}$ Ingerod, Brastad, Sweden, formerly Special Programme for Research and Training in Tropical Diseases (TDR), World Health Organization, Geneva, Switzerland

${ }^{3}$ Center for Mental Health Research College of Health and Medicine, Australian National University, Canberra, Australian Capital Territory, Australia

${ }^{4}$ Center of Emergency Medical Services, Mashhad University of Medical Sciences, Mashhad, Iran

\section{Correspondence}

Mahmood Tara, Department of Medical Informatics, Faculty of Medicine, Mashhad University of Medical Sciences, Mashhad, Iran.

Email: taram@mums.ac.ir

Funding information

Mashhad University of Medical Sciences, Grant/Award Number: 970861

\section{Summary}

Objective: This study aimed to develop an age-included approach to measure the potential accessibility to Emergency medical services (EMS) across urban and suburban areas of Mashhad city in Iran.

Methods: We used an improved version of two-step floating catchment area (2SFCA) and enhanced 2SFCA (E2SFCA) methods to measure the potential accessibility to EMS services with inclusion of age factor. This enabled us to better model accessibility of the older population to the EMS. We used 22800 records of EMS enquiries from July to September 2018 to evaluate and assess the potential improvement in access to the EMS with incorporating age in the model.

Results: Suburb areas had less potential accessibility compared with central urban due to the high density of EMS stations in the city center areas. Our model showed slight improvement in potential accessibility measurement as a result of the proposed age-integrated method.

Conclusion: An age-integrated index of accessibility method takes into account the age distribution of the population in service area is highly associated with actual measures of accessibility to EMS services. Identifying areas with poor access to EMS will help policymakers to design better policy 
planning to allocate resources and improve provision of EMS services.

\section{KEYWORDS}

2SFCA, ambulance, emergency medical services, Iran, spatial accessibility

\section{1 | BACKGROUND}

Emergency medical services (EMS), also known as ambulance services, provide medical transport and/or out-ofhospital medical care to people at incidents or to patients who need emergency treatment. ${ }^{1}$ Nowadays, the demand for EMS in large cities has increased due to population growth, urban violence, aging population, a growing number of people with sedentary lifestyles, and the occurrence of chronic diseases. ${ }^{2}$ Increased demand for prehospital services will reduce accessibility of people to EMS, and this leads to increased mortality and morbidity rates of people in need of health services. ${ }^{3,4}$ Therefore, health care policies should be updated to keep up with these changes.

Access to health care services can be divided into two broad categories: Actual accessibility and potential accessibility. ${ }^{5}$ Approaches which measure the former method use real-world data. For example, the actual response time of ambulances (time from call to a vehicle arriving at the scene) could be a proxy for actual accessibility to EMS. ${ }^{6}$ Potential accessibility is an indicator of the ability of individuals to use this service. ${ }^{7}$ For example, the computed driving time between a patient's residence and the closest ambulance service is an indicator of potential accessibility. It could be low, but the response time is much higher in practice because of inaccessibility of the closest ambulance, traffic, and issues related to the dispatch system. ${ }^{8}$ So, in many cases the potential accessibility is estimated to be higher than the actual accessibility.

Potential accessibility to health care services is a multidimensional issue, including appropriate supply (availability), reasonable travel distance/time to available services (proximity), level and nature of need for those seeking care (health needs), and the ability of individuals to access care at the time of need (mobility). ${ }^{9,10}$ The first two dimensions are often referred to as "spatial accessibility" with the latter ones referred to as "nonspatial" dimensions. One area could have a good potential spatial access if the availability of services were adequate and located at reasonable proximity. However, a population with a higher level of health needs require more services to maintain an equivalent level of access compared with an average population of similar size with equal potential spatial access. The mobility of a population is another important factor determining the ability of people to overcome the distance barrier. ${ }^{11}$ If these nonspatial factors incorporated in accessibility models, the computed potential accessibility could become closer to the actual accessibility.

Measuring the potential spatial accessibility is a common approach to measure access to health care services. ${ }^{12,13}$ Various measures of spatial accessibility have been proposed, including regional availability, ${ }^{14}$ the gravity model, ${ }^{15}$ and the two-step floating catchment area (2SFCA) method. ${ }^{16}$ The regional availability method is the simplest which computes the ratio of supply and demand within a predefined area. However, it does not reveal the spatial variation within the boundary, nor does it account for the interaction between supply and demand across the boundary. The gravity model is theoretically more sound but requires more computation and interpretation of the result is not intuitive. $^{17,18}$

The 2SFCA method, a special case of the gravity approach, uses supply and demand to find potential spatial accessibility. This method was widely used in other studies to show accessibility to health care facilities. ${ }^{19-21}$ It has drawn sharp criticism because it assumes that all the population locations within a catchment area have equal accessibility, while it disregards differences in accessibility within the catchment. This is known as distance decay in the 
literature. ${ }^{22,23}$ To address this issue, many authors have tried to improve the 2SFCA method. ${ }^{24-26}$ One of these attempts, conducted by Luo and Qi (2009), presents the enhanced two-step floating catchment area (E2SFCA) method. As the name suggests, it accounts for distance decay by assigning different weights associated with different travel time zones. ${ }^{24}$ On the other hand, some studies have tried to improve the 2SFCA method by integrating nonspatial factors. ${ }^{11,20,27}$ For example, McGrail et al (2009) proposed an index of rural access to primary care by integrating health needs and mobility within the 2SFCA methodology, ${ }^{28}$ while Wang et al (2005) showed accessibility to primary care facilities by integrating spatial and nonspatial factors when defining health professional shortage areas. $^{27}$

Although the literature already includes a considerable number of studies which measured access to various health care facilities such as primary health care, ${ }^{12,22,29}$ access to EMS ambulances is rarely discussed. ${ }^{3}$ Availability and quality of data and visualization technology such as Geographical Information Systems (GIS), have made identification of underserved areas more feasible.. ${ }^{23}$ In this study, we aimed to assess potential accessibility of EMS ambulances in the city of Mashhad using 2SFCA and E2SFCA methods by considering age as an important nonspatial factor in EMS utilization. The proposed methods were compared with response time (as a proxy of actual accessibility) to show how much potential access results could be similar to reality.

\section{2 | METHODS}

Study area:

Mashhad is the capital city of Razavi Khorasan and the second most populated city in Iran. It covers an area of $350 \mathrm{~km}^{2}$ and has over three million population in urban areas.. ${ }^{30}$ As shown in Figure 1, Mashhad has 50 EMS stations with 71 ambulances and five road stations that provide EMS in city area.

Approach:

The present study was conducted in three phases as follows:

Phase 1: Data collection

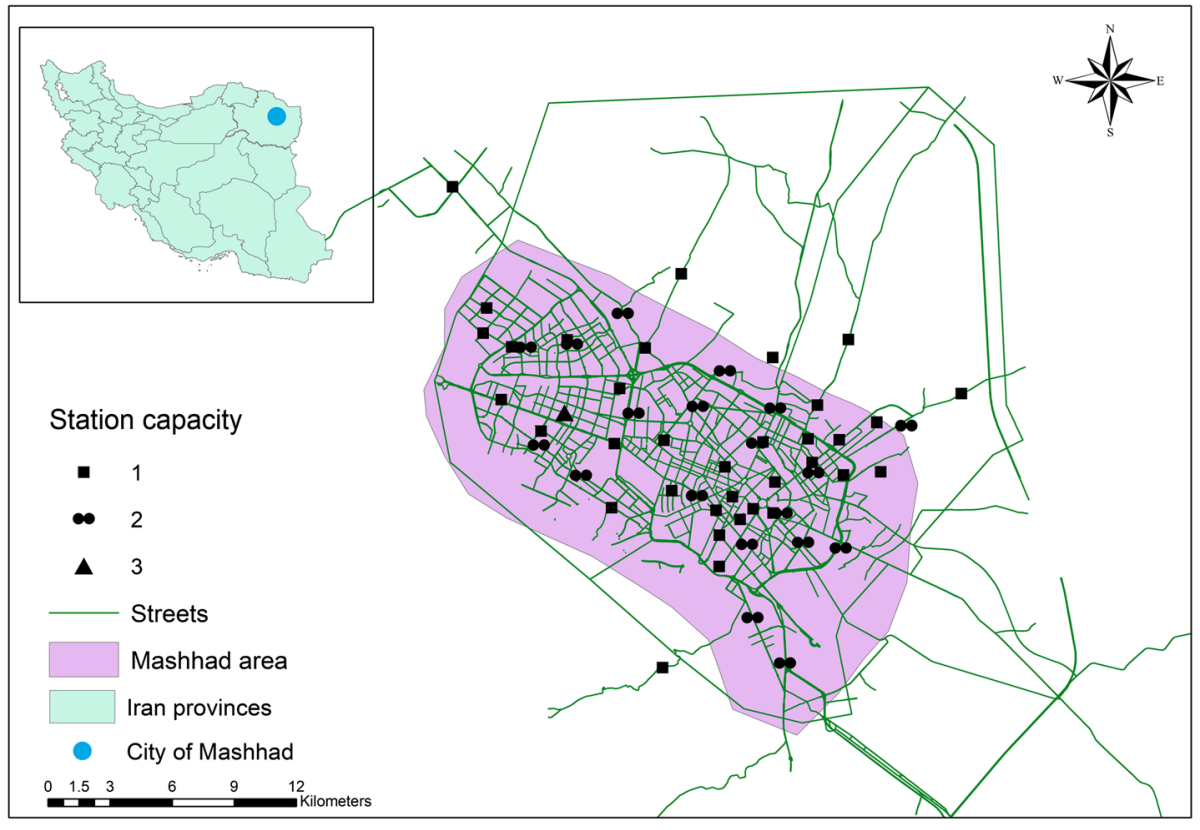

FIGURE 1 EMS stations distribution and their capacity (number of ambulances for each station) in Mashhad 
We obtained 2016 census data from Mashhad Municipality Council, consisting of 17000 census blocks (the smallest census unit in Iran) and 141 districts. Information on gender and age distribution was available for each census block. EMS call data of 2018, including data on patient age and mission times, were obtained from the City Emergency Management Center. In addition, callers' locations were available in latitude and longitude format ( $X, Y$ coordinate) only from July to September 2018.

Phase 2: Measuring potential accessibility

For each census block, the centroid location was calculated by geometry tool in ArcGIS package (ESRI, Redlands, CA, USA). Travel-time buffer for each census block centroid and EMS stations location were estimated with the ArcMap network analysis module. Based on the different road classes in the road network, the standard speed was set at $80 \mathrm{~km} / \mathrm{h}$ for expressways, $60 \mathrm{~km} / \mathrm{h}$ for main roads, $50 \mathrm{~km} / \mathrm{h}$ for secondary roads, and $30 \mathrm{~km} / \mathrm{h}$ for other roads. To estimate the potential accessibility, we used four methods as follows:

Method 1: 2SFCA

This approach was used to capture the interactions between ambulance availability and the population distribution generating an access score for each of census blocks through a two-step process:

Step 1: For each EMS station $j$, we searched all population locations $(k)$ within a threshold travel time $\left(d_{0}=10\right.$ min) from station $j$ (ie, the catchment of this EMS location) and computed the provider-to-population ratio $R_{j}$ within the catchment area by Equation (1):

$$
R_{j}=\frac{S_{j}}{\sum_{k \in\left\{d_{k j} \leq d_{0}\right\}} P_{k}}
$$

where $P_{k}$ is the population at location $k$ where the centroid falls within catchment $j\left(d_{k j} \leq d_{0}\right), S_{j}$ the number of ambulances at station $j$, and $d_{k j}$ the travel time between $k$ and $j$.

Step 2: For each census block centroid $i$, we searched all station locations $(j)$ within the threshold travel time $\left(d_{0}=\right.$ $10 \mathrm{~min}$ ) from location $i$ (ie, the catchment area) and summed up the provider-to-population ratios (derived in step 1) $R_{j}$ at these locations by Equation (2):

$$
A_{i}^{F}=\sum_{j \in\left\{d_{i j} \leq d_{0}\right\}} R_{j}
$$

where $A_{i}^{F}$ represents the accessibility of the population at block $i$ to EMS ambulances based on the 2SFCA method, $R_{j}$ the provider-to-population ratio at station location $j$ where the centroid falls within the catchment area centered at population location $i$ (ie, $d_{i j} \leq d_{0}$ ), and $d_{i j}$ is the travel time between $i$ and $j$.

Method 2: Enhanced 2SFCA

The 2SFCA method did not consider distance decay effect in measuring accessibility index; this leads to unrealistic estimate of access scores. To overcome this limitation of 2SFCA, we employed the E2SFCA method introduced by Luo et al (2009) and deployed in our setting as follows:

Step 1: Three catchments of station location $j$ were set to 5,10 , and 15-minute driving time, for zones 1 to 3 , respectively. We searched all population locations $(k)$ that were within a threshold travel time zone $\left(D_{r}\right)$ from location $j$ (ie, catchment area $j$ ), and computed the weighted provider-to-population ratio $R_{j}$ within the catchment area using Equation (3):

$$
R_{j}=\frac{S_{j}}{\sum_{k \in\left\{d_{k j} \in D_{r}\right\}} P_{k} W_{r}}=\frac{S_{j}}{\sum_{k \in\left\{d_{k j} \in D_{1}\right\}} P_{k} W_{1}+\sum_{k \in\left\{d_{k j} \in D_{2}\right\}} P_{k} W_{2}+\sum_{k \in\left\{d_{k j} \in D_{3}\right\}} P_{k} W_{3}}
$$

where $P_{k}$ is the part of the population of census block $k$ falling within the catchment $j\left(d_{k j} \in D_{r}\right), S_{j}$ the number of ambulances at station $j, d_{k j}$ the travel time between $k$ and $j$, and $D_{r}$ the $r^{\text {th }}$ travel time zone $(r \in\{1,2,3\})$ within the 
catchment. $W_{r}$ is the distance weight for the $r^{\text {th }}$ travel time zone calculated from the Gaussian function. The weight set $\{1.00,0.68,0.22\}$ was used for capturing the distance decay of access to the station $j$.

Step 2: For each population of location $i$, we searched all station locations ( $j$ ) within the 15-minute travel time zone from location $i$ (ie, catchment area i) and summed up the provider-to population ratios (calculated in step 1) $R_{\mathrm{j}}$ at these locations by Equation (4):

$$
A_{i}^{F}=\sum_{j \in\left\{d_{i j} \leq D_{r}\right\}} R_{j} W_{r}=\sum_{j \in\left\{d_{i j} \in D_{1}\right\}} R_{j} W_{1}+\sum_{j \in\left\{d_{i j} \in D_{2}\right\}} R_{j} W_{2}+\sum_{j \in\left\{d_{i j} \in D_{3}\right\}} R_{j} W_{3}
$$

where $A_{i}^{F}$ represents the accessibility for the population at location $i$ to EMS, $R_{j}$ the provider-to-population ratio at station location $j$ that falls within the catchment centered at population $i\left(i e, d_{k j} \in D_{r}\right.$ ), and $d_{i j}$ the travel time between $i$ and $j$. The same distance weights derived from the Gaussian function used in step 1 were applied to different travel time zones to account for the distance decay.

Method 3: Integrating age distribution with 2SFCA

To integrate age as a significant factor of health needs in the EMS setting, we performed weighting of each age category in the population in the accessibility formula to find the relative age effect on EMS access score. If an age category in a population spells a higher risk (eg, older age group), a higher weight should be used for that population. We obtained weights for each age category based on incidence in the list of call data. For this purpose, we used call data of a whole year (2018) to find the rate of EMS requests for each age group. As shown in Table 1, the number of emergency call provided by the City Emergency Management Center and people in each age group provided by Mashhad Municipality Council were used ${ }^{30}$ to calculate weights as a proxy of emergency needs for each age group.

By integrating the age weights with 2SFCA method, the accessibility index could be updated as shown below:

Step 1: For each EMS station $j$, we searched all population locations $(k)$ that were within a threshold travel time $\left(d_{0}\right.$ $=10 \mathrm{~min}$ ) from station $j$ (ie, the catchment of this EMS location) and calculated weighted population by multiplying associated weights to number of population in each age group. Then the weighted provider-topopulation ratio $R_{j}$ within the catchment area was calculated by Equation (5):

$$
R_{j}=\frac{S_{j}}{\sum_{k \in\left\{d_{k j} \leq D_{0}\right\}} \sum_{n \in\{G\}} P_{k n} W_{n}}
$$

where $G$ is the number of age groups, $P_{k n}$ the population of age group $n$ at location $k$ where the centroid falls within catchment $j\left(d_{k j} \leq d_{0}\right), S_{j}$ the number of ambulances at station $j$, and $d_{k j}$ the travel time between $k$ and $j$.

TABLE 1 Rate of EMS requests of Mashhad in each age group

\begin{tabular}{|lccc|}
\hline Age Group & Number of EMS Calls in 2018 & Population & Weight $=$ rate $\times 100$ \\
\hline "0-9" & 10488 & 616761 & 1.70 \\
\hline "10-19" & 20799 & 483789 & 4.30 \\
\hline "20-29" & 41973 & 614173 & 6.83 \\
\hline "30-39" & 38928 & 673313 & 5.78 \\
\hline "40-49" & 27667 & 416852 & 6.64 \\
\hline "50-59" & 25989 & 294613 & 8.82 \\
\hline "60-69" & 20899 & 159972 & 13.06 \\
\hline "70-79" & 17400 & 77533 & 22.44 \\
\hline $80+$ & 14177 & 35654 & 39.76 \\
\hline
\end{tabular}


Step 2: For each census block centroid $i$, we searched all station locations $(j)$ within the threshold travel time $\left(d_{0}=\right.$ $10 \mathrm{~min}$ ) from location $i$ (ie, catchment area $i$ ) and summed up the provider-to-population ratios (derived in step 1) $R_{j}$ at these locations as shown in Equation (6):

$$
A_{i}^{F}=\sum_{j \in\left\{d_{i j} \leq d_{0}\right\}} R_{j}
$$

where $A_{i}^{F}$ represents the accessibility of population at block $i$ to EMS ambulances, $R_{j}$ the provider-to-population ratio at station location $j$ whose centroid falls within the catchment centered at population location $i$ (ie, $d_{i j} \leq d_{0}$ ), and $d_{i j}$ the travel time between $i$ and $j$.

Method 4: Integrating age distribution with E2SFCA

This method represents a compounded use of both methods no. 2 and no. 3 . We used both weights of different travel zones and age groups to calculate the accessibility measure.

After applying these four methods (to reach a better interpretation and comparison of results), the averages of access scores of census blocks in each district were calculated and visualized. We used natural break method to classify the indices in five class.

Phase 3: Measuring actual access and comparison

We used the call data from the city EMS stations for three months (July to September 2018) to measure actual accessibility. Response time for each call and location of events were extracted from EMS system database. We spatially joined the call locations to each district area and calculated the average response times as actual accessibility indices for each district. Next, the results of potential accessibility were compared with actual accessibility by Pearson's correlation statistic using SPSS, version 16. Finally, we calculated the nearest station for each caller's location using the ArcMap network analysis tool to evaluate to which degree dispatches were optimized.

\section{3 | RESULTS}

Table 2 demonstrates characteristics of EMS calls from July to September 2018 in Mashhad. From 22800 patients who received care, $52 \%$ were male. The mean age was 43.2 years old, and mean response time of the missions was 12.31 minutes; $71.8 \%$ of calls were due to medical issues, and $28.2 \%$ were for traumatic incidents. Only $36.8 \%$ of calls serviced from the nearest station to the scene, and $63.2 \%$ serviced from farther stations.

TAB LE 2 Characteristics of EMS calls from July to September, 2018

$\begin{array}{ll}\text { Characteristics } & \text { All Patients }(\mathbf{n}=\mathbf{2 2} 800) \\ \text { Sex (\%) } & 15351(52.2) \\ \text { Male } & 12428(44.8) \\ \text { Female } & 43.2(22.0) \\ \text { Mean age (SD) } & 12.31(6.02) \\ \text { Mean response time in minute (SD) } & 16371(71.8) \\ \text { Incidence type } & \text { 16) } \\ \text { Medical } & 6429(28.2) \\ \text { Trauma } & \\ \text { Serviced station } & 8391(36.8) \\ \text { Nearest station to the scene } & 14409(63.2) \\ \text { Farther stations to the scene } & \end{array}$


Figure 2 shows the potential accessibility to EMS services in Mashhad districts with the different access measures. In 2SFCA method, red color signifies areas where there were less than 17 ambulances accessible for one million residents within 10 minutes; in yellow areas, access score is greater than red areas, and it is nearly doubled. However, for age-integrated methods, the interpretation of the accessibility index is not easy as 2SFCA and E2SFCA because age-integrated methods use age weighted measure for population. All maps highlight that peripheral areas in Mashhad city have low access to EMS.

Figure 3 shows actual access in Mashhad districts. Patients living in the areas shown in yellow color had better accessibility to the EMS services ( $<9 \mathrm{~min}$ ) compared with those who are living in areas highlighted in red color ( $>13.5$ $\mathrm{min}$ ). In overall, actual accessibility in the city center is low compared with potential accessibility.

The association between potential accessibility indices and actual accessibility (real world repose time) is assessed using Pearson correlation matrix. The correlation coefficients were negative, both for the potential accessibility indices and the actual accessibility index. Correlation coefficient is a measure of the strength and direction of the linear relationship between two variables. It assumes values in the range from -1 to +1 , where +1 indicates the

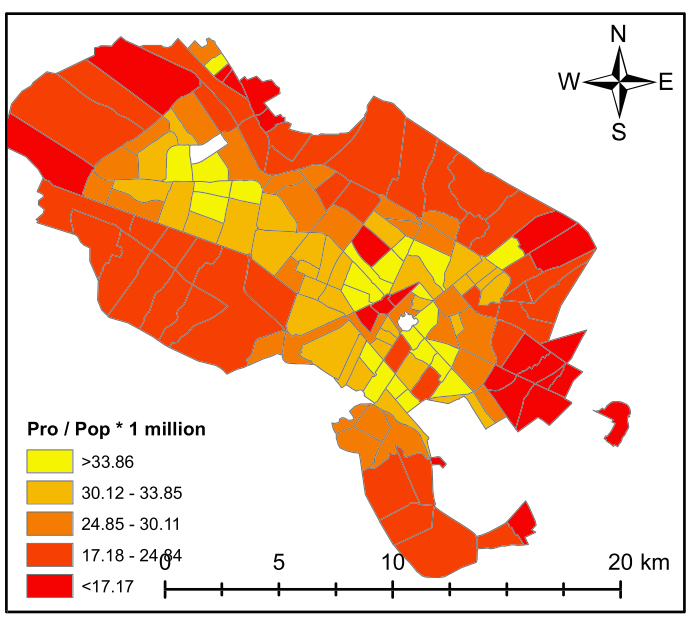

(A)

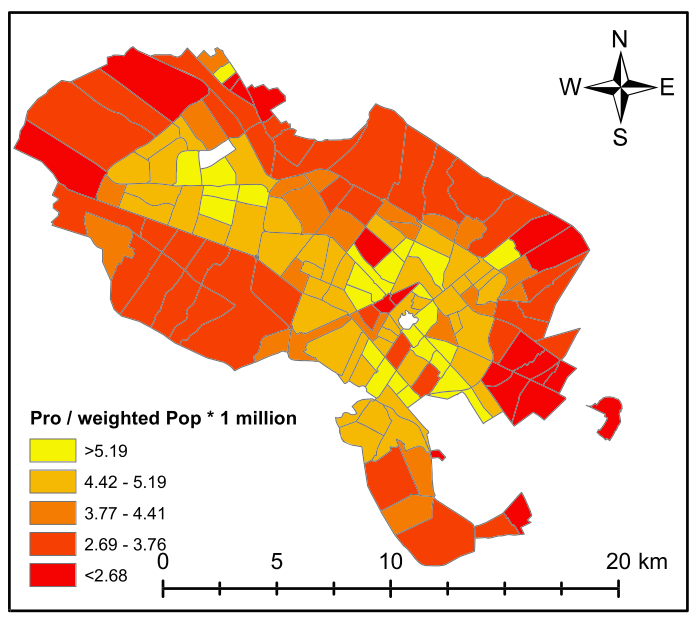

(C)

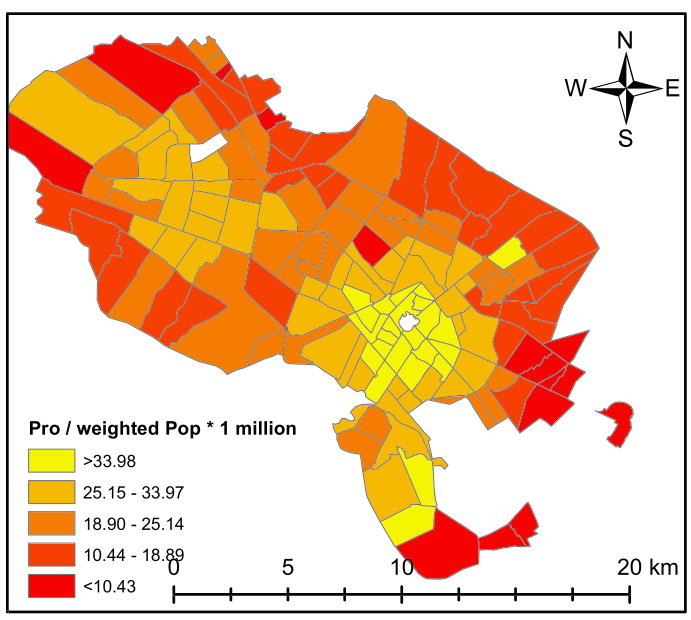

(B)

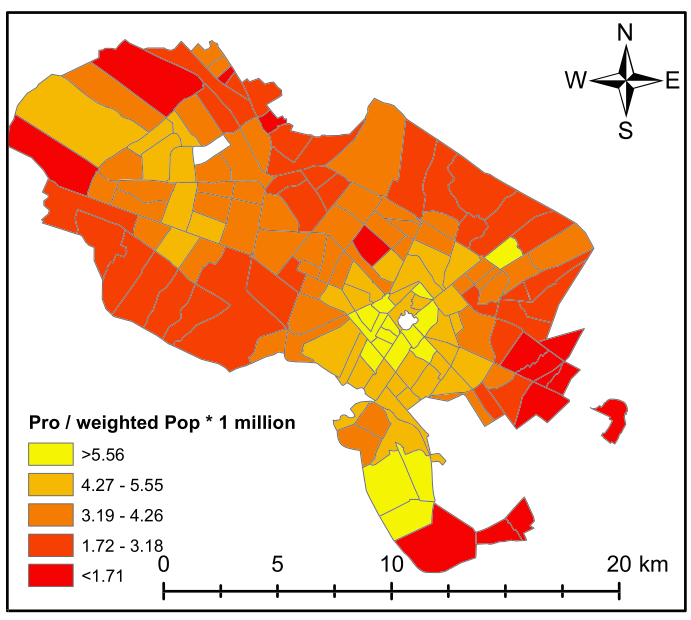

(D)

FIGURE 2 Potential access to EMS services in the Mashhad districts. (A) Two-step floating catchment area (2SFCA); (B) enhanced 2SFCA; (C) age-integrated 2SFCA; and (D) age-integrated enhanced 2SFCA 


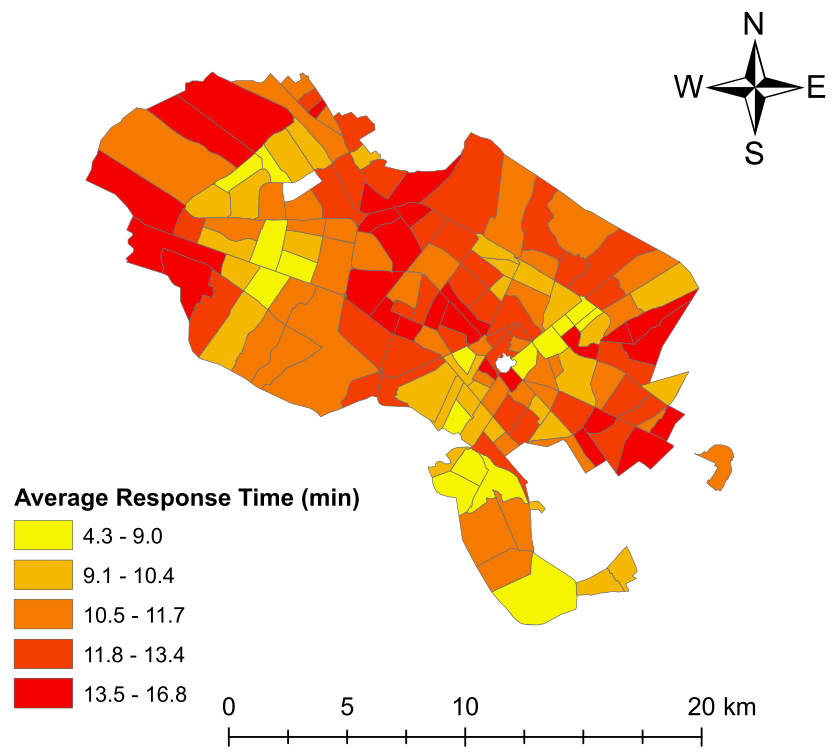

FIGURE 3 Actual access index in Mashhad districts

strongest possible agreement in the same direction, -1 indicates the strongest possible agreement in the opposite direction, and 0 shows no agreement between two measures. In this study, we used it to measure the relationship between the four methods with actual real-world data. Although the coefficients did not indicate a strong correlation, it improved when the enhanced model with the integrated age factor was applied (Table 3).

\section{DISCUSSION}

The emphasis of this research was on developing approaches to measure the potential accessibility of EMS in an urban area based on the 2SFCA method. This study revealed that using enhanced 2SFCA and integrating age distribution can improve the accuracy of potential accessibility index (Table 3). In most of the time, it is not possible to measure actual accessibility, especially in developing countries without high-tech EMS systems. However, wherever such systems are available, implementation in each urban area should help policymakers to allocate appropriate services for enhancing patient accessibility to EMS. The results of this study identified some areas with poor EMS accessibility in border regions of Mashhad City. Therefore, appropriate distribution of EMS services is significant in narrowing the inequity gaps in EMS access for the study area.

To our knowledge, this is the first study attempting to model potential EMS accessibility of services in a large city with lower to middle income economy status. ${ }^{30}$ A study by Tansley et al (2009) measured EMS accessibility in Ghana at the country level rather than at small area level (eg, census blocks). However, some attempts have been made to integrate nonspatial factors in the accessibility index in other research areas. For example, Bagheri et al (2009) and McGrail et al (2009) both integrated a need index for measuring accessibility to primary health care facilities.

Researchers should pay attention to the fact that the EMS setting constitutes a situation where there is a critical difference compared with other contexts. In the EMS setting, ambulances come to patients' locations delivering care instead of patients themselves visiting the medical centres. This is the reason that many nonspatial factors such as

TAB LE 3 Correlation coefficient of potential access indices and actual access

\begin{tabular}{llccc} 
& 2SFCA & E2SFCA & Age-Integrated 2SFCA & Age-Integrated E2SFCA \\
\hline Response time (actual access) & $-0.240^{*}$ & $-0.258^{*}$ & $-0.286^{*}$ & $-0.309^{*}$
\end{tabular}

${ }^{*}$ Pearson correlation coefficient is significant ( $P$ value $\left.<.005\right)$. 
mobility $^{31}$ of patients and public transportation ${ }^{32}$ are not applicable to the EMS setting. Consequently, we considered only the age distribution of population as a nonspatial factor in the present study.

The authors are aware that even a perfect potential spatial access in a particular area would not necessarily lead to better access in reality due to many other confounding factors. However, this study showed that among the four methods investigated, the age-integrated E2SFCA method showed better results compared with actual accessibility in emergency settings (Table 3). As it can be seen in this table, all potential accessibility indices had negative correlations with actual accessibility index. This result seems reasonable because average response times of missions are lower in regions with a higher accessibility index. As shown in Table 2, 63.2\% of callers were not serviced from the nearest station which could significantly affect the response time when expressed as actual accessibility index. Ideally, if all of requests were serviced from the nearest station, the results shown in Table 3 might have been considerably improved. We suggest that policymakers could investigate the ambulance efficiency and also apply some spatial models to determine if the distribution of ambulances within the study area is optimized.

Our study has some limitations. First, our network dataset was simple, and there was no traffic information available for our study area. The central part of city suffers from traffic congestion more than the border parts, and this may be the reason that the central parts had a better potential accessibility index compared with actual access. The second limitation was the accuracy of call data. Critical data like the time reaching the scene were not automatically stored using the global positioning system, and these data are recorded manually by the ambulance staff. This may generate random error and may affect our results. Thirdly, we extracted the population of each block from census data which did not consider the potential of travelers arriving from other cities. Additionally, we did not have any data estimating the flow of residents during day and night.

\section{5 | CONCLUSION}

Access to health care services, and particularly EMS services, will continue to be an important challenge especially in low income countries. It is then important for the authorities in charge to employ methods that measure accessibility of such services in a way that equity of service provision across all the population in need is carefully visited. In this research, we improved 2SFCA method, as one of the most-commonly used methods of spatial accessibility measurement, by inclusion of age factor as one of the deterministic factors in need of assessment of EMS service allocation. Our finding showed that such enhancement could be more associated with actual accessibility. In other words, our research showed that in areas where the population is older, there is a need for more ambulance services. Therefore, any policy intervention on EMS resource allocation should consider age-included approach to accessibility measurement.

\section{ACKNOWLEDGEMENT}

We would like to thank the staff at Mashhad EMS center.

\section{CONFLICT OF INTEREST}

The authors declare that they have no conflict of interest.

\section{FUNDING SOURCE}

This project is funded by Mashhad University of Medical Sciences (grant number 970861) 


\section{ETHICAL APPROVAL}

This project is approved by ethical committee of Mashhad University of Medical Sciences (number 970861; date 12/5/2018)

\section{ORCID}

Soheil Hashtarkhani (1D) https://orcid.org/0000-0001-7750-6294

\section{REFERENCES}

1. Moore L. Measuring quality and effectiveness of prehospital EMS. Prehosp Emerg Care. 1999;3(4):325-331.

2. Nogueira LC Jr, Pinto LR, Silva PM. Reducing Emergency Medical Service response time via the reallocation of ambulance bases. Health care management science. 2016;19(1):31-42.

3. Tansley G, Stewart B, Zakariah A, et al. Population-level spatial access to prehospital care by the national ambulance service in Ghana. Prehosp Emerg Care. 2016;20(6):768-775.

4. Peleg K, Pliskin JS. A geographic information system simulation model of EMS: reducing ambulance response time. American Journal of Emergency Medicine. 2004;22(3):164-170.

5. Kiani B, Bagheri N, Tara A, Hoseini B, Tabesh H, Tara M. Revealed access to haemodialysis facilities in northeastern Iran: factors that matter in rural and urban areas. Geospatial Health. 2017;12(2):237-244.

6. Mahmud A, Aljunid SM. Availability and accessibility of subsidized mammogram screening program in peninsular Malaysia: a preliminary study using travel impedance approach. PloS one. 2018;13(2):e0191764.

7. Hansen WG. How accessibility shapes land use. Journal of the American Institute of planners. 1959;25(2):73-76.

8. Dean SF. Why the closest ambulance cannot be dispatched in an urban emergency medical services system. Prehospital and disaster medicine. 2008;23(2):161-165.

9. Aday LA, Andersen R. A framework for the study of access to medical care. Health services research. 1974;9(3):208-220.

10. Penchansky R, Thomas JW. The concept of access: definition and relationship to consumer satisfaction. Medical care. 1981;19(2):127-140.

11. McGrail MR, Humphreys JS. The index of rural access: an innovative integrated approach for measuring primary care access. BMC Health Serv Res. 2009;9:124.

12. Langford M, Higgs $G$. Measuring potential access to primary healthcare services: the influence of alternative spatial representations of population. The Professional Geographer. 2006;58(3):294-306.

13. Ni J, Wang J, Rui Y, Qian T, Wang J. An enhanced variable two-step floating catchment area method for measuring spatial accessibility to residential care facilities in Nanjing. International journal of environmental research and public health. 2015;12(11):14490-14504.

14. Khan AA. An integrated approach to measuring potential spatial access to health care services. Socio-economic planning sciences. 1992;26(4):275-287.

15. Joseph AE, Bantock PR. Measuring potential physical accessibility to general practitioners in rural areas: a method and case study. Social science \& medicine. 1982;16(1):85-90.

16. Luo W, Wang F. Measures of spatial accessibility to health care in a GIS environment: synthesis and a case study in the Chicago region. Environment and Planning B: Planning and Design. 2003;30(6):865-884.

17. Joseph AE, Phillips DR. Accessibility and utilization: geographical perspectives on health care delivery: Sage; 1984.

18. Luo W, Whippo T. Variable catchment sizes for the two-step floating catchment area (2SFCA) method. Health \& place. 2012;18(4):789-795.

19. Luo W. Using a GIS-based floating catchment method to assess areas with shortage of physicians. Health \& place. 2004;10(1):1-11.

20. Bagheri N, Benwell GL, PGDipsci M. Modelling accessibility to primary health care using a spatial accessibility index and a need index. Hawai 'IJ Pub Health. 2008;1:14.

21. Cervigni F, Suzuki Y, Ishii T, Hata A. Spatial accessibility to pediatric services. Journal of Community Health. 2008;33(6): 444-448.

22. McGrail MR, Humphreys JS. Measuring spatial accessibility to primary health care services: utilising dynamic catchment sizes. Applied Geography. 2014;54:182-188.

23. Vadrevu L, Kanjilal B. Measuring spatial equity and access to maternal health services using enhanced two step floating catchment area method (E2SFCA)-a case study of the Indian Sundarbans. International journal for equity in health. 2016;15(1):87. 
24. Luo W, Qi Y. An enhanced two-step floating catchment area (E2SFCA) method for measuring spatial accessibility to primary care physicians. Health \& place. 2009;15(4):1100-1107.

25. Wan N, Zou B, Sternberg T. A three-step floating catchment area method for analyzing spatial access to health services. International Journal of Geographical Information Science. 2012;26(6):1073-1089.

26. Polzin P, Borges J, Coelho A. An extended kernel density two-step floating catchment area method to analyze access to health care. Environment and Planning B: Planning and Design. 2014;41(4):717-735.

27. Wang F, Luo W. Assessing spatial and nonspatial factors for healthcare access: towards an integrated approach to defining health professional shortage areas. Health \& place. 2005;11(2):131-146.

28. McGrail MR, Humphreys JS. A new index of access to primary care services in rural areas. Australian and New Zealand journal of public health. 2009;33(5):418-423.

29. McGrail MR, Humphreys JS. Measuring spatial accessibility to primary care in rural areas: improving the effectiveness of the two-step floating catchment area method. Applied Geography. 2009;29(4):533-541.

30. [Available from: https://www.citypopulation.de/php/iran-admin.php?adm2id=0916.

31. Bisht SS, Mishra V, Fuloria S. Measuring accessibility for inclusive development: a census based index. Social Indicators Research. 2010;98(1):167-181.

32. Mao L, Nekorchuk D. Measuring spatial accessibility to healthcare for populations with multiple transportation modes. Health \& place. 2013;24:115-122.

How to cite this article: Hashtarkhani S, Kiani B, Bergquist R, Bagheri N, VafaeiNejad R, Tara M. An ageintegrated approach to improve measurement of potential spatial accessibility to emergency medical services for urban areas. Int J Health Plann Mgmt. 2020;1-11. https://doi.org/10.1002/hpm.2960 